\title{
Los protocolos de actuación con víctimas de acoso escolar. Análisis de una normativa institucional
}

\author{
Fuensanta Cerezo \\ Universidad de Murcia
}

\begin{abstract}
Resumen: Las medidas de protección ante la violencia entre escolares iniciadas a partir de los informes del Defensor del Pueblo, deben promover normativas de actuación y la Ley Reguladora de la Responsabilidad Penal de los Menores (5/2000) establece la justicia de la restauración, que obliga a resarcir el daño causado, premisa debe ser tenida en cuenta en las normativas. Este trabajo analiza si las propuestas institucionales de la Comunidad de Murcia (España) sobre victimización escolar (Protocolos AVE) se ajustan a estas necesidades. Se seleccionaron los protocolos específicos y se procedió al análisis de contenido por descriptores y fases de actuación. En cuanto descriptores, el acosador no está incluido en las actuaciones específicas y la restitución no se contempla. El psicólogo es asumido por el orientador. Los directores son responsables en primera instancia y el equipo de orientación es asesor. Las fases de actuación: detección, intervención con implicados y sus familias y seguimiento. Para la atención al acosador remite al documento donde se hace un listado de medidas, en su mayoría sanciones punitivas. Indican la necesidad de actuar en defensa de las víctimas, pero las propuestas no concretan efectivos, acciones de protección, cambio de actitudes, ni tampoco la restitución.
\end{abstract}

Palabras clave: Victimización escolar, bullying, protocolos, prevención, intervención.

\section{The protocols for acting with bullying victims. Analysis of an institutional normative}

\begin{abstract}
The protection measures against school violence (bullying) initiated based on the Ombudsman' reports must promote acting regulations. The Law Regulating the Criminal Responsibility of Minors (5/2000) establishes the justice of restitution, which dictates making amends for the harm caused, a premise that must be taken into account in the regulations. This paper analyzes whether the institutional proposals of the Community of Murcia (Spain) for action against school victimization (AVE Protocols) meet these needs. Specific protocols were selected and content analysis was carried out by descriptors and action phases. Regarding the descriptors, the bully is not included in the specific actions, and restitution is not contemplated. The psychologist's role is assumed by the guidance counselor. The principals are responsible in the first instance, and the guidance team as advisor. The phases of action are: detection, intervention with those involved and their families, and follow-up. Regarding attention to the bully, it refers to a list of measures, mostly punitive sanctions. The need to act in defense of the victims is indicated, but the proposals do not specify who controls these situations, which actions of protection and change of attitudes should be carried out, or how to perform the restitution.

Keywords: School victimization, bullying, protocols, prevention, intervention.
\end{abstract}

Desde diversas áreas educativas relacionadas con la infancia y la adolescencia se viene alertando de altas tasas de violenta entre los escolares. En España, los informes

Recibido: 18/06/2018 - Aceptado: 19/04/2019 - Avance online: 07/05/2019 *Correspondencia: Fuensanta Cerezo.

Universidad de Murcia.

C.P: 30100 , Murcia, España.

E-mail:fcerezo@um.es realizados por el Defensor del Pueblo, el último publicado en 2007, y más reciente las investigaciones de UNICEF (2014), indican que es necesario articular normas y ajustar responsabilidades con los participantes, tanto sobre el alumnado como con el profesorado, que promuevan la convivencia pacífica y consigan la inclusión efectiva de todos en las aulas. A partir de las indicaciones propuestas, 
las diferentes administraciones públicas han ido promulgando normativas sobre convivencia en las diferentes comunidades autónomas, con el objetivo de facilitar al profesorado estrategias de actuación ante este problema. Pero también desde el Ministerio de Educación y desde la Fiscalía de Menores se han publicado instrucciones y normativas legales como la Ley Orgánica 1/1996 (modificada 29 de julio de 2015) que sirven de marco legal para actuar ante los casos de bullying y ciberbullying. Así, en 2005 se publica la Instrucción 10/2005, de la Fiscalía General del Estado, que recoge los procedimientos de actuación ante el acoso escolar. En febrero de 2007 se publica el Real Decreto 275/2007, por el que se crea el Observatorio Estatal de la Convivencia Escolar, y más reciente, la Resolución de 31 03-2015, para el fomento de la convivencia y la erradicación de la violencia.

Las Administraciones de las distintas Comunidades Autónomas han promulgando distintos decretos y protocolos de actuación para el profesorado, basados en las directrices generales señaladas desde la administración central, proponiendo medidas de prevención y actuación ante el acoso escolar, normas para la promoción de la paz y la convivencia e incluso espacios como la creación de las aulas de convivencia (Alonso, EscuderoVidal, De Castro y Castaño-Calle, 2017; Cerezo y Rubio, 2018).

En la Ley Reguladora de la Responsabilidad Penal de los Menores (5/2000), se establece como punto imprescindible, la justicia de la restauración. Esto implica que todo protocolo de actuación debe incluir medidas que faciliten la restitución a la víctima y no solo medidas coercitivas y correctoras.

Las premisas anteriores indican que, las propuestas de las administraciones deberían responder a cuestiones como las siguientes:

1. El bullying es una forma de interacción frecuente y recurrente entre escolares (Garaigordobil, 2011) también en su forma virtual o ciberbullying (Arnaiz, Cerezo, Giménez y Maquilón, 2016); no precisa de provocación, y cuyo objetivo fundamentalmente "divertirse" (Cerezo, 2014 ; Zych, Ortega-Ruiz, y Del Rey, 2015). Esta relación se sustenta en un desequilibrio de poder donde impera la ley del más fuerte apoyada por el silencio cómplice de los observadores (Salmivalli, 2009). Por tanto, la primera cuestión que debe responder sería si se puede justificar el bullying como una forma de interacción consentida (DíazAguado, Martínez y Martín, 2013), ya que solamente con la conciencia de "tolerancia cero" podremos asegurar su no ocurrencia.

2. En segundo lugar, plantear qué fortalece a los agresores, qué recompensas reciben (Simon \& Nail, 2013). Realmente, existe un potencial de gratificación intrínseca y por tanto es preciso reconocer qué se esconde tras las acciones violentas y ahí, probablemente haya que cuestionar cómo son las relaciones entre los miembros de la comunidad educativa (García-Bacete et al., 2013; Wang et al., 2016) y dejar de circunscribirlo exclusivamente a los protagonistas directamente implicados.

3. El punto anterior nos conduce a declarar que la escuela, como espacio de convivencia, debe intervenir ante cualquier sospecha de acoso, es su responsabilidad prioritaria favorecer la convivencia y la inclusión de todo el alumnado (Cerezo, Ruiz-Estaban, Sánchez y Arense, 2018; Ortega, Del Rey y Casas, 2013). En este sentido, los estudios alertan de que un estilo educativo represor autoritario puede favorecer comportamientos intolerantes entre sus miembros (Prodócimo, Cerezo y Arense, 2014; Wang et al., 2016) y alejarles de la cooperación necesaria para el reconocimiento de cada miembro como parte del grupo (Cerezo, 2014). Por tanto, la escuela ante los casos de violencia relacional, debe asumir parte de responsabilidad civil y penal junto con las familias de los menores (Cerezo y Rubio, 2018).

4. Pero, la actuación sancionadora suele generar estrategias de evitación y no estrategias de cooperación, como sería deseable (Ttofi y Farrington, 2008). De hecho, 
el número de denuncias por acoso escolar ha aumentado considerablemente en los últimos años, lo que nos lleva a cuestionar la eficacia de las diferentes normativas generales, así como las normas de convivencia específicas elaboradas en cada centro escolar (Cerezo, 2016). Esto subraya que un listado de conductas contrarias a las normas y sus correspondientes sanciones no es suficiente para erradicar la violencia, e incluso que puede tener un efecto contrario al esperado.

El objetivo de este trabajo es analizar las propuestas institucionales de la Comunidad de Murcia para la actuación ante la victimización escolar. Todas ellas se recogen en los protocolos AVE (Protocolos de apoyo inmediato y coordinado a las víctimas escolares), publicados en 2015.

\section{MÉTODO}

\section{MUESTRA}

La normativa recoge siete protocolos: 1. Atención a alumnado víctima de agresión aislada; 2. Atención a estudiantes objeto de acoso escolar; 3 . Intervención con víctimas de accidentes, emergencias o catástrofes; 4. Actuación escolar en situaciones de enfermedad de media o larga duración del alumnado; 5. Gestión del duelo en los centros docentes; 6. Intervención escolar ante actos autolíticos; y 7 . Atención a víctimas de conductas contra la libertad e indemnidad sexual. Se seleccionaron los protocolos relacionados con la temática y se procedió al análisis de contenido por descriptores y fases de actuación.

En cuanto a su contenido, según se declara en la introducción, estos documentos pretenden abordar "las diferentes situaciones conflictivas que podrían suceder en un centro educativo" (CARM. Protocolos AVE, 2015: 3). Indica que ésta será la normativa vigente en relación a las problemáticas tratadas y propone las primeras intervenciones a tener en cuenta, tanto dirigidas a los alumnos directamente afectados como a sus familias. Su objetivo principal es servir de esquema para las intervenciones ante los problemas de violencia escolar, desde las actuaciones previas que con carácter preventivo pudieran realizarse a las que corresponda cuando se produzca una situación de emergencia. Hace especial mención al carácter privado y a la necesidad de garantizar la protección efectiva de los derechos de los alumnos en situación de riesgo o vulneración y violación de derechos, evitando prácticas revictimizadoras.

\section{PROCEDIMIENTO}

\section{Criterios de selección}

Los documentos seleccionados para este trabajo corresponden a los protocolos 1 y 2 de la publicación de la CARM que lleva por título Protocolos AVE, Versión 1 : 16/02/2015.

Protocolo 1. Atención al alumnado víctima de agresión aislada. Se activará en situaciones de agresión aislada que conlleven daño físico o psicológico. Tras la detección, se intervendrá con el agredido, con los compañeros del grupo aula y las familias. Siempre respetando la confidencialidad.

Protocolo 2. Atención al alumnado objeto de acoso escolar. El objetivo principal es facilitar al centro pautas de actuación, para ello establece una serie de indicaciones en las fases de detección, actuación con el acosado, como por ejemplo adoptar medidas de protección, seguridad y apoyo expreso o indirecto; con los compañeros, el profesorado y las familias.

\section{Descriptores analizados}

Teniendo en cuenta la literatura comentada en la Introducción, se seleccionaron los siguientes descriptores: Acosador/agresor, Acoso, Bullying, Ciberbullying, Familia/ res, Orientador/a, Prevención, Psicólogo/a, Restitución/restituir, Víctima. Además se analizaron las distintas fases de intervención que recogen estos protocolos: Detección, intervención y seguimiento. 


\section{RESULTADOS}

\section{ANÁLISIS POR DESCRIPTORES}

Al introducir los descriptores encontramos lo siguiente:

- Acosador/agresor. En la descripción del problema, pero no en las actuaciones. "También resulta fundamental determinar los alumnos implicados como presuntos agresores y víctimas, así como posibles alumnos observadores no participantes en la agresión" (CARM. Protocolos AVE, 2015: 8)

- Acoso: en repetidas ocasiones.

- Bullying: No en el texto, si en las referencias.

- Ciberbullying. No aparece.

- Familia/res. Son elemento clave en todas las fases.

- Orientador/a se recoge como asesor en todas las fases del proceso.

- Prevención. Solo aparece una vez: "Implicar a los alumnos en la creación de un marco protector, preventivo y correctivo de la soledad, aislamiento y victimización" (CARM. Protocolos AVE, 2015: 18)

- Psicólogo/a. No aparece.

- Restitución/ Restituir. No aparece.

- Víctima. Como sujeto de las agresiones.

\section{ANÁLISIS POR FASES}

Vemos que establece dos categorías de acoso: "agresión aislada" y "acoso escolar", promulgando protocolos específicos para cada una. En ambos protocolos, tras una definición propone tres momentos o fases de intervención (Figura 1):

1. Fase de detección: análisis e información preliminar. En esta fase se tratará de determinar si existe el problema, quienes están implicados y quiénes son los observadores.

2. Fase de intervención: Se prestará atención urgente y asesoramiento. Con el grupo de alumnos se proponen estrategias como la mediación escolar y los métodos cooperativos. Asimismo se informará a las familias.

3. Fase de seguimiento: Se valorará la necesidad de apoyos expertos externos. En cada fase se indica quienes deben actuar: Comunidad, Director, Orientador, Tutor.

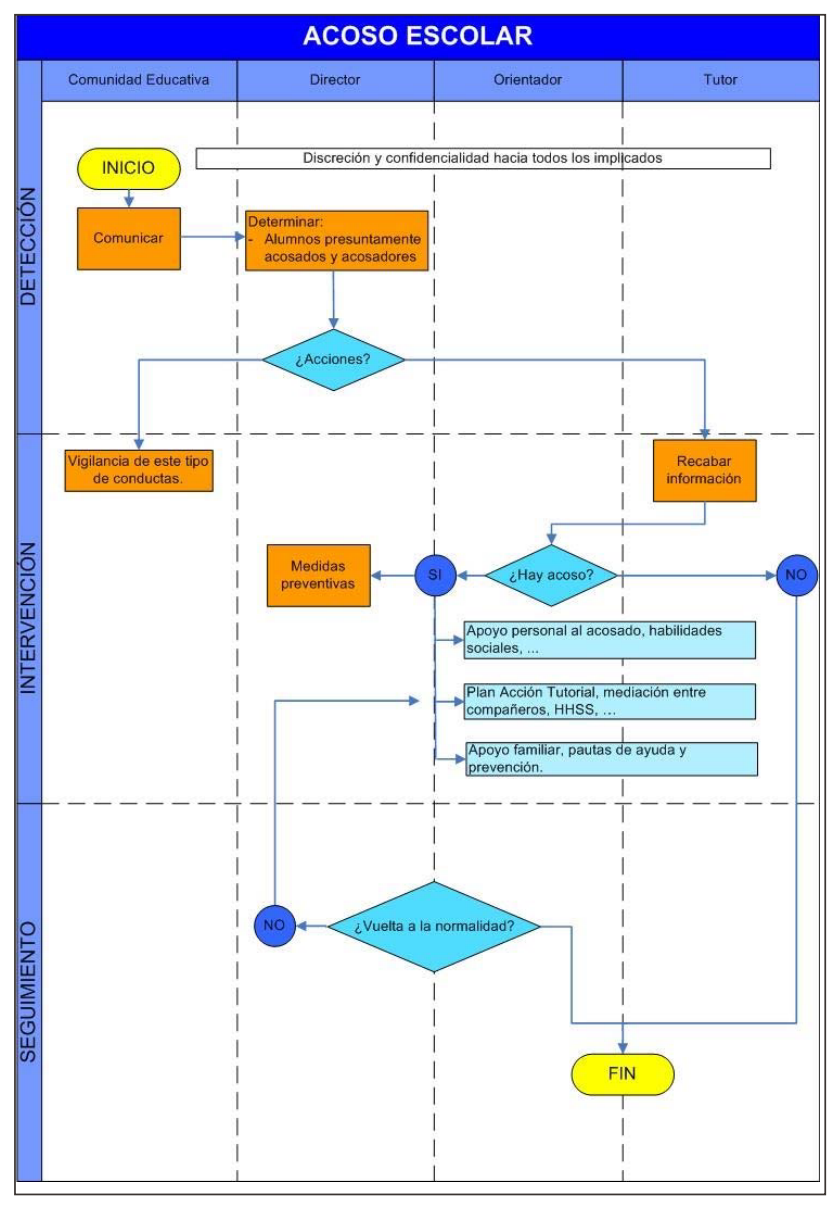

Figura 1. Flujograma de actuación. (Tomado de CARM, Protocolos AVE, 2015: 15).

\section{DISCUSIÓN}

Los resultados indican que los protocolos plantean la necesidad de actuar en defensa de las víctimas, comenzando por una fase de detección del problema.

En cuanto al contenido, los protocolos definen qué se entiende por agresión puntual y la diferencia del acoso escolar, estableciendo diferentes estrategias, principalmente según la gravedad del caso. Cabe destacar la importancia que se concede a preservar la intimidad de la víctima y a facilitarle apoyo. Sin embargo, para la atención al acosador, no se establecen estrategias conjuntas (Ortega, Del Rey y Casas, 2013) y remite al documento donde se hace un listado de medidas, en su mayoría sanciones punitivas que van desde la amonestación a la expulsión del centro, acciones que, como se ha apuntado, pueden generar un alto rechazo a la escuela y fomentar la consolidación de conductas contrarias a la convivencia (GarcíaBacete et al., 2013; Ttofi y Farrington, 2008). 
Los protocolos indican quiénes deben actuar, señalando a los directivos como responsables en primera instancia y al equipo de orientación como asesor, contando con la intervención del Tutor. Mientras que la figura del experto, que puede entenderse como el psicólogo, solo se contempla como estrategia externa, cuando las consecuencias del problema desborden las capacidades de los educadores, siendo un recurso humano de gran trascendencia en estos conflictos (Alonso et al., 2017). Además, en estos protocolos no se hace mención a la necesidad de formación específica para los profesionales de la educación que facilite la detección e intervención ante la violencia entre los escolares (Cerezo, Ruiz-Esteban, Sánchez y Arense, 2018).

Se establecen diferentes fases de actuación con las víctimas, desde la detección, intervención con los implicados, el aula y sus familias y el seguimiento, lo que está en consonancia con lo propuesto por los expertos.

Podemos concluir que las propuestas que indican los protocolos de actuación, tanto ante la agresión "aislada" como ante el acoso escolar, además de ser muy generales, no se ajustan a los esperado, ya que no concretan personal, ni efectivos para controlar estas situaciones, ni cómo llevar a cabo la restitución del daño causado, tampoco aborda lo que es más importante, cómo plantear su prevención, ya que se actúa cuando ya se detecta el problema, y no propone estrategias concretas de trabajo en el aula para la mejora de la convivencia.

\section{- Conflicto de intereses.}

Los autores declaran no tener ningún conflicto de intereses.

\section{REFERENCIAS}

AA.W. (2007). Violencia escolar. El maltrato entre iguales en la ESO. Madrid: Oficina del Defensor del Pueblo.

Alonso, F., Escudero-Vidal, J., De Castro, R., \& Castaño-Calle, R. (2017). Convivencia y paz en el Derecho Educativo español. Congreso sobre Derecho Educativo del curso 2015 2016. Actas al congreso. pp.47-75.
Arnaiz, P., Cerezo, F., Giménez, A. M., \& Maquilón, J. J. (2016). Conductas de ciberadicción y experiencias de cyberbullying entre adolescentes. Anales de Psicología, 32(3), 761-769. doi:10.6018/ analesps.32.3.217461

CARM. Consejería de Educación Región de Murcia (2015). Protocolos AVE. Recuperado de http://www.carm.es

Cerezo, F. (2014). Diferencias en estatus social entre roles en bullying. Un análisis sociométrico. Bordón, 66(2), 33-46. doi:10.13042/Bordon.2014.66202

Cerezo, F. (2016). ¿̇Son útiles los programas de intervención? ¿Cómo evaluar su eficacia? En J. L. Castejón (Coord.). Psicología y Educación: Presente y Futuro (pp. 92-99). Alicante: ACIPE.

Cerezo, F., \& Rubio, F.J. (2018). Medidas relativas al acoso escolar y ciberacoso en la normativa autonómica española. Un estudio comparativo. Revista Electrónica Interuniversitaria de Formación del Profesorado REIFOP, 20(1), 113-126. doi:10.6018/reifop/20.1.253391

Cerezo, F., Ruiz-Esteban, C., Sánchez, C., y Arense, J.,J. (2018). Dimensions of parenting styles, social climate, and bullying victims in primary and secondary education. Psicothema, 30(1), 59-65. doi:10.7334/ psicothema2016.360

Díaz-Aguado, M. J., Martínez, R., \& Martín, J. (2011). El acoso escolar en España. Prevalencia, papeles adoptados por todo el grupo a características a las que atribuyen la victimización. Revista de Educación, 362 (348-380). doi: 10.4438/1988-592X-RE2011-362-164.

Garaigordobil, M. (2011). Prevalencia y consecuencias del cyberbullying: una revisión. International Journal of Psychology and Psychological Therapy, 17 (2), 233-254.

García-Bacete, F. J., Jiménez, I., Muñoz, M. V., Monjas, M. I., Sureda, I., Martín, L. J., Marande, G., y Sanchiz, M. L. (2013). Aulas como contextos de aceptación y apoyo para integrar a los alumnos rechazados. Apuntes de Psicología, 31 (2), 11 -13.

UNICEF (2014). La infancia en España2014. El valor social de los niños: hacia un Pacto de 
Estado por la Infancia. Recuperado de www. unicef.es.

Ortega, R., Del Rey, R., \& Casas, J. A. (2013). La Convivencia Escolar: clave en la predicción del Bullying. Revista Iberoamericana de Evaluación Educativa, 6(2), 91-102.

Prodócimo, E., Cerezo, F., \& Arense, J. J. (2014). Acoso escolar: variables sociofamiliares como factores de riesgo o de protección. Behavioral Psychology / Psicología Conductual, 22(2), 343-357.

Salmivalli, C. (2009). Bullying and the peer group: A review. Aggression and Violent Behavior, 15, 112-120

Simon, J. B., \& Nail, P. R. (2013) Introduction to special issue on bullying: A social influence perspective, Social Influence, 8, 2-3, 81-86. doi:10.1080/15534510.2013.771882
Ttofi, M. M., \& Farrington, D. P. (2008). Bullying: short-term and long-term effects, and the importance of defiance theory in explanation and prevention. Victims and Offenders, 3, 289-312.

Wang, C., Swearer, S. M., Lembeck, P., Collins, A., \& Berry, B. (2015) Teachers Matter: An Examination of Student-Teacher Relationships, Attitudes Toward Bullying, and Bullying Behavior. Journal of Applied School Psychology, 37 (3), 219-238. doi:10.1080/ 15377903.2015.1056923

Zych. I., Ortega-Ruiz, R., \& Del Rey, R. (2015). Systematic review of theoretical studies on bullying and cyberbullying: Facts, knowledge, prevention, and intervention. Aggression and Violent Behavior, 23, 1-21. 